\title{
Evaluation ex ante de la prévision saisonnière climatique en petit paysannat burkinabé
}

\author{
W.P.I. Dabire ${ }^{1,2}{ }^{*}$ B. Barbier ${ }^{3,4}$ N. Andrieu ${ }^{5,6}$
}

\begin{abstract}
Mots-clés
Programmation linéaire - Aide à la décision - Saison - Prévision Pluviométrie - Climat - Gestion du risque - Burkina Faso.
\end{abstract}

\begin{abstract}
Résumé
La production agricole de I'Afrique sub-saharienne essentiellement pluviale est particulièrement vulnérable à la variabilité climatique. A l'échelle des exploitations, cette variabilité a un impact direct sur la production primaire, sur le revenu et la sécurité alimentaire de la famille. Dans cette étude, nous évaluons l'intérêt économique de mettre la prévision de la pluviométrie d'hivernage à la disposition des producteurs. Cette étude a été menée dans la zone de Dano au sud-ouest du Burkina Faso. Elle repose sur l'utilisation d'un modèle de programmation linéaire qui maximise le revenu en optimisant l'allocation des terres, du travail et des intrants entre plusieurs types de cultures, les rendements variant selon le type de sol, d'itinéraire technique et d'hivernage. Quatre scénarios ont été analysés : un scénario témoin où le paysan n'a pas accès aux prévisions, un scénario où les producteurs ont accès à une prévision d'hivernage $\mathrm{sec}$, un autre où ils ont accès à une prévision d'hivernage normal et un dernier où ils ont accès à une prévision d'hivernage humide. Les résultats des simulations montrent que $c^{\prime}$ est une prévision d'hivernage sec qui présente le plus d'intérêt mais c'est aussi celui où l'erreur de prévision serait la plus coûteuse. Globalement le gain de revenu de la prévision est relativement peu élevé, mais le coût d'une erreur de prévision pose le problème des responsabilités et des compensations. Ces résultats relativisent l'intérêt des prévisions saisonnières pour la réduction de la vulnérabilité des populations des pays sahéliens.
\end{abstract}

\section{INTRODUCTION}

Les producteurs d'Afrique sub-saharienne sont depuis longtemps confrontés à une forte variabilité climatique (1) face à laquelle ils ont développé une gamme importante de stratégies d'adaptation. Mais avec l'augmentation annoncée de l'occurrence des phénomènes climatiques extrêmes, la recherche doit proposer des outils de décision innovants aux producteurs pour limiter leur incertitude face à

\footnotetext{
1. Institut de l'environnement et de recherches agricoles, département Gestion des ressources naturelles / Système de production, BP 8645, Ouagadougou, Burkina Faso. 2. Université de Ouagadougou, UFR en Sciences économiques et de gestion, Ouagadougou, Burkina Faso.

3. Centre de coopération internationale en recherche agronomique pour le développement, UMR G-eau, 34398 Montpellier, France.

4. Institut international d'ingénierie de l'eau et de l'environnement, Ouagadougou, Burkina Faso.

5. Cirad, UMR Innovation, 34398 Montpellier, France.

6. Centre international de recherche-développement sur l'élevage en zone subhumide, Bobo-Dioulasso, Burkina Faso.

* Auteur pour la correspondance

Tél. : +2267647 7716 ; fax : +22650307242

E-mail : warvarp@yahoo.fr
}

la variabilité climatique. La prévision saisonnière de la pluviométrie constitue l'un de ces outils et de nombreux auteurs ont montré son intérêt potentiel pour la prise de décision des producteurs d'Afrique sub-saharienne $(2,3,4,5)$. Néanmoins, les effets potentiels d'une erreur de prédiction ont rarement été quantifiés. La région Sud-Ouest du Burkina Faso est la plus favorable à la production de biomasse agricole du pays mais est marquée par une forte variabilité spatiotemporelle des précipitations ayant des répercussions sur les choix de production et les revenus des producteurs. Ingram et coll. (3) ont montré que, dans cette zone, mettre à la disposition des agriculteurs les données de prévision saisonnière permet d'améliorer leurs choix de production, mais ces auteurs n'ont pas quantifié l'impact économique des prévisions et le coût d'une erreur de prévision.

L'objectif de cette étude a été d'évaluer l'intérêt de la prévision saisonnière dans le sud-ouest du Burkina Faso. Cette prévision saisonnière concerne la pluviométrie d'hivernage. Deux hypothèses ont été formulées : dans la première, mettre à la disposition des producteurs une prévision d'hivernage humide procure le meilleur gain en leur permettant de mettre en place des cultures à fort potentiel de production. Dans la deuxième, le coût d'une erreur de prévision est plus important que le gain d'une bonne prévision. 
A partir d'un modèle de programmation linéaire, nous avons comparé à un scénario témoin trois scénarios où les producteurs d'un bassin versant du Burkina Faso ont accès à différentes modalités de prévision saisonnière et nous avons quantifié l'effet d'une erreur de prévision sur le revenu. Après avoir présenté la zone d'étude puis les caractéristiques du modèle utilisé, nous analysons les résultats des différents scénarios, puis discutons de l'intérêt de la prévision saisonnière et des limites de la méthode proposée.

\section{MATERIEL ET METHODES}

\section{Zone d'étude}

Cette étude a été menée dans le sud-ouest du Burkina Faso, dans le village de Pontieba, commune de Dano (6) (figure 1). Il s'agit d'une zone relativement enclavée limitant les possibilités de diversification des activités extra-agricoles. La pluviométrie moyenne est de $850 \mathrm{~mm}$ et l'hivernage s'étend de mai à octobre. Il est suivi d'une saison sèche froide de novembre à fin décembre et d'une saison sèche chaude de janvier à mai.

Les systèmes de production rencontrés dans la zone sont essentiellement basés sur la polyculture. Les systèmes de cultures présentent des résistances contrastées à la sécheresse. Ces cultures sont de plus localisées sur des types de terres très variés : les bonnes terres qui sont profondes et situées en bas de pente, les terres plus marginales, les bas-fonds aménagés, les bas-fonds non aménagés inondés en hivernage, les bas-fonds non aménagés peu inondables et enfin les périmètres irrigués. Les principales cultures pluviales rencontrées dans la zone sont, par ordre d'importance, le sorgho, le mil, le coton, le maïs et, dans une moindre mesure, le niébé, l'arachide, le sésame. Le sorgho, le coton et parfois le maïs sont cultivés en rotation sur les bonnes terres, le mil sur les terres marginales et le maïs autour des cases. Le niébé est le plus souvent cultivé en association avec une céréale. Dans les bas-fonds inondés en hivernage, les principales cultures sont le riz et le sorgho. Dans les bas-fonds peu inondables on peut aussi trouver du maïs. Sur les périmètres irrigués, les paysans cultivent du riz en hivernage, et en saison sèche du riz et des cultures légumières.

Les jachères pratiquées dans la zone sont de courte durée. La fertilisation des terres dépend des quantités de fumure organique apportées. Les apports d'engrais minéraux, encore faibles, sont liés à la culture du coton, puisque la compagnie cotonnière distribue à crédit des intrants (engrais et pesticides) dont le coût sera déduit des recettes générées à l'issue de la récolte. On observe une progressive apparition des pratiques de conservation des sols, telles

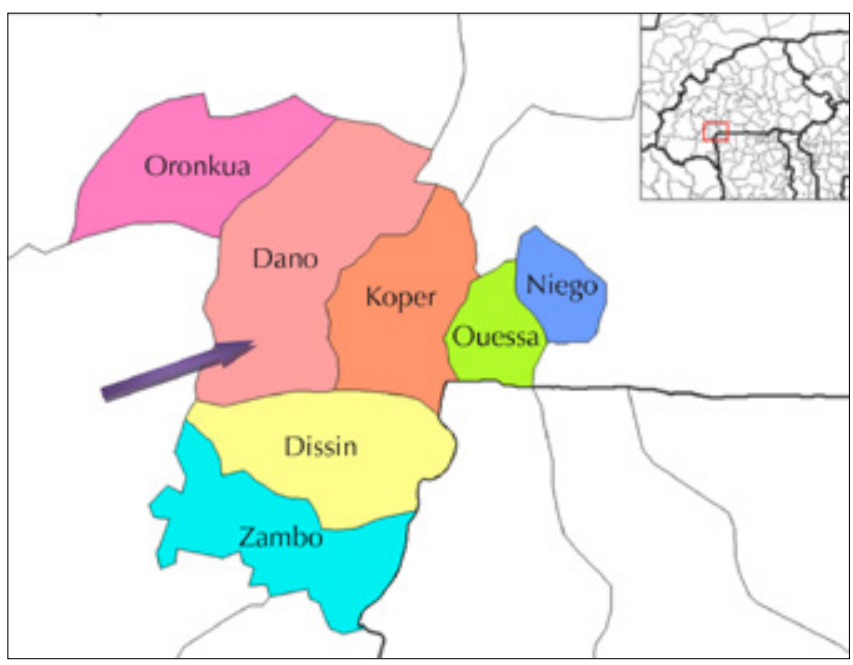

Figure 1 : localisation de la commune de Dano au Burkina Faso. que la construction de cordons pierreux, de diguettes, de bandes herbacées et la plantation de haies vives. La présence de maraîchage sur un périmètre non clôturé limite le développement de l'élevage. Certaines exploitations possèdent néanmoins quelques petits ruminants pouvant jouer un rôle d'épargne.

\section{Echantillonnage et collecte des données}

Une enquête sur un échantillon de cent ménages du village de Pontieba choisis de manière aléatoire a été effectuée en deux phases dans le but de décrire les activités menées par les agriculteurs, leurs contraintes, et de générer des données pouvant permettre de paramétrer le modèle à construire (14). Dans la première phase, les caractéristiques structurelles des exploitations de la zone ont été répertoriées. Dans la seconde phase, l'enquête a porté sur l'utilisation des terres, notamment grâce à l'identification des parcelles à l'aide d'un système de positionnement global (GPS).

Le périmètre irrigué de Pontieba couvre environ 25 hectares, soit environ 2 p. 100 de la surface du bassin versant, et le réservoir situé en amont peut contenir $300000 \mathrm{~m}^{3}$. Il permet en cas de bon remplissage, l'irrigation de cultures successives, généralement du riz pendant l'hivernage, et deux cultures de contre-saison, généralement du riz, du maïs ou de la tomate. La seconde culture de contre-saison et parfois la première culture de contre-saison ne sont pas assurées, la taille du bassin versant ne permettant pas de remplir le réservoir chaque année. La surface irriguée dépend donc du remplissage du bassin qui lui dépend des pluies de l'hivernage.

\section{Formulation du modèle}

Le modèle utilisé dans la présente étude s'inscrit dans la tradition de la modélisation bioéconomique qui vise à représenter les interactions entre un stock de capital naturel et l'activité économique dont il est le support (7). Dans le cas présent, il s'agit du stock d'eau dans le réservoir. La programmation mathématique permet de décrire les différentes activités élémentaires que les agriculteurs du bassin versant peuvent mener et qui génèrent des produits et consomment des ressources. Nous avons fait le choix de ne pas représenter les systèmes d'élevage compte tenu de la forte orientation agricole des systèmes étudiés. De plus, pour les systèmes d'élevage, la saison d'hivernage n'est pas la saison la plus critique, l'offre fourragère et les réserves hydriques étant alors abondantes. Nous avons également choisi de ne pas représenter les activités extra-agricoles compte tenu du relatif enclavement de la zone. Les contraintes propres à l'exploitation, telles que les surfaces disponibles pour les différents types de cultures et la force de travail présente, sont fournies en entrée au modèle. Par un processus d'optimisation, le programme recherche la meilleure combinaison d'activités qui permet d'atteindre les objectifs de l'exploitant, décrits par une fonction des différents produits de ces activités. Cette fonction objectif est, dans notre cas, un surplus monétaire annuel de tout le bassin versant que le programme cherche à maximiser. Le revenu monétaire total issu de l'exploitation du bassin à maximiser se calcule ainsi :

$$
\begin{aligned}
& \sum_{c}[\operatorname{VE}(c) \times p x v(c)]-\sum_{c}[X(c, s) \times c s e m(c)+X(c, s) \times \operatorname{int}(c, s)] \\
& -\sum_{c}[A C(c) \times p a(c)]-[\text { cred } \times \text { taux }]-[\text { pxo } \times \text { terre }(\text { irr })]
\end{aligned}
$$

Autrement dit, il s'agit de soustraire des quantités des produits vendus $V E$ par culture $c$ multipliées par le prix unitaire de vente $\operatorname{pxv}(c)$ de chaque culture, les coûts des semences $\operatorname{csem}(c)$ et des autres intrants int $(c, s)$ par unité de surface cultivée multipliés par la surface des cultures $X(c, s)$, moins les achats de céréales $A C(c)$ pour la consommation que multiplie un coefficient de préférence alimentaire 
pour chaque céréale $p a(c)$, moins les frais financiers liés aux crédits de campagne multipliés par le montant du crédit contracté Cred, moins les redevances pxo par unité de surface irriguée terre(irr).

Les activités du modèle sont les cultures comptées en hectares. Nous avons seulement considéré les cultures pures, plus largement pratiquées dans la zone. Pour le maïs en particulier et conformément aux pratiques observées dans la zone d'étude, nous avons distingué trois niveaux d'intensification : extensif, moyennement intensif et très intensif.

Le modèle distingue les bonnes terres utilisées en pluvial (s1), les terres marginales utilisées en pluvial (s2), les terres irriguées en hivernage (s3), les terres irriguées en saison sèche froide (s4) et les terres irriguées en saison sèche chaude (s5). En outre, on distingue deux types de bas-fonds, les bas-fonds peu inondés plus favorables au maïs (s6), et ceux inondés plus favorables au riz et au sorgho (s7).

Le modèle distingue également trois types d'hivernage, un sec, un humide et un normal défini à dire d'experts. A chaque type d'hivernage pour chaque culture et par type de terre correspond un niveau de rendement $r d t(c, s, h)$, des temps de travaux et des niveaux d'utilisation d'intrants spécifiques déterminés à partir des enquêtes de terrain et de la connaissance des systèmes de production de la zone (14).

A chaque type d'hivernage est associée une probabilité d'occurrence proba(h). Pour calculer la production $P A(c)$ de chaque culture par type de terre (et pour chaque niveau d'intensification dans le cas du maïs), le modèle réalise la somme des productions calculées pour chaque type d'hivernage et modulées par leur coefficient d'occurrence spécifique.

$$
\sum_{s} \sum_{h} X(c, s) \times r d t(c, s, h) \times \operatorname{proba}(h)=P A(c)
$$

De même, pour chaque type d'hivernage, le prix des cultures vivrières varie, mais dans des directions inverses à celle des rendements. Ainsi, à un hivernage sec sont associés des rendements bas et des prix élevés et inversement pour un hivernage humide, sauf pour le coton dont seuls les rendements varient, les prix étant fixés. La volatilité des prix des cultures vivrières, de même que l'impact d'une erreur de prévision sur les prix des denrées ne sont pas analysés dans cette étude.

Le modèle est constitué des contraintes traditionnelles : terre, travail et capital. Nous avons ajouté une contrainte de consommation de céréales car les paysans consomment une bonne partie de leur production. Nous avons rajouté une contrainte d'eau liée au périmètre irrigué et une contrainte de risque dans la mesure où certains choix des paysans s'expliquent par leur aversion au risque.

Le paramètre terre(s) désigne les surfaces des différents types de terre, $s$ étant le type de terre. La variable surface en culture est représentée par $X(c, s)$; la somme des superficies cultivées doit être inférieure ou égale à la superficie disponible dans tout le bassin versant par type de terre:

\section{$\sum_{c} \sum_{s} X(c, s) \leq \operatorname{terre}(s)$}

La campagne agricole a été divisée en six périodes pour tenir compte plus spécifiquement de la contrainte travail : p1 l'installation des cultures pluviales, p2 la récolte des cultures pluviales, p3 l'installation des cultures irriguées de la saison sèche froide, $\mathrm{p} 4$ la récolte des cultures irriguées de la saison sèche froide, $\mathrm{p} 5$ l'installation des cultures irriguées de la saison sèche chaude et p6 la récolte des cultures irriguées de la saison sèche chaude. La détermination de la quantité de travail nécessaire tn durant les six périodes agricoles et selon le type de culture prend en compte le nombre de jours de travail disponible $t d$ par personne multiplié par le nombre de personnes dans le bassin pop multiplié par la proportion d'actifs dans la population $c \_$pers :

$$
\sum_{c} \sum_{s} X(c, s) \times t n(c, p) \leq t d \times \text { pop } \times c_{-} \text {pers }
$$

Nous avons défini le paramètre Bes_cap $(c, s)$ qui désigne le besoin en capital par hectare de culture et par type de terre pour l'achat des semences $\operatorname{csem}(c)$ et des intrants $\operatorname{int}(c, s)$ :

$\operatorname{Bes\_ cap}(c, s)=\operatorname{csem}(c)+\operatorname{int}(c, s)$

Le modèle offre la possibilité de recourir au crédit de campagne cred, cap étant un paramètre désignant le capital dont dispose l'exploitation :

$$
\sum_{c} \sum_{s} X(c, s) \times B e s_{-} c a p(c, s) \leq c a p+c r e d '
$$

Le crédit de campagne dans la zone est lié à la surface en coton (cot) et au besoin en intrant d'un hectare de coton :

$$
\sum_{c} \sum_{s} X(\cot , s) \times B e s \_c a p(\cot , s) \geq c r e d '
$$

Pour tenir compte de la contrainte en eau sur le périmètre irrigué de Pontieba, nous avons intégré les paramètres q_eau(s), qui désigne la quantité d'eau disponible dans le réservoir, et Bes_ $e a u(c, s)$ le besoin en eau (en $\mathrm{m}^{3}$ par ha de cultures). L'équation cidessous distingue les six périodes de l'année et le surplus d'eau d'une période $O_{s-1}(p)$ est transféré à la période suivante moins un pourcentage dû à l'évaporation :

$$
\sum_{c} \sum_{s} X(c, s) \times \text { Bes_eau }(c, s)+O_{s}(p) \leq q_{-} e a u(s)+O_{s-1}(p)
$$

La contrainte de consommation de céréales stipule que le ménage doit couvrir ses besoins en céréales cons qui est un paramètre désignant le besoin en céréales en kilogramme par personne et par an multiplié par la population pop. La variable $A U(c)$ est la quantité autoconsommée de céréales, la variable $A C(c)$ la quantité optimale de céréales éventuellement achetée pour la consommation du ménage et $p a(c)$ un coefficient de préférence alimentaire pour chaque céréale. La contrainte alimentaire s'écrit alors :

$$
\sum_{c}(A U(c)+A C(c)) \times p a(c) \geq \text { cons } \times \text { pop }
$$

La contrainte du risque stipule que le revenu d'une année défavorable ne doit pas être inférieur à un revenu minimum rmin. La variable rmin sert à calibrer le modèle, c'est-à-dire que rmin est ajusté de manière exogène jusqu'à ce que le modèle reproduise ce que les paysans font réellement.

$$
\begin{aligned}
& \sum_{c}[V E(c) \times p x v(c)]-\sum_{c}[X(c, s) \times c s e m(c)+X(c, s) \times \operatorname{int}(c, s)] \\
& -\sum_{c}[A C(c) \times p a(c)]-[\operatorname{cred} \times \operatorname{taux}]-[\text { pxo } \times \text { terre }(\text { irr })] \geq r \min
\end{aligned}
$$

\section{Scénarios testés}

Les scénarios proposés visent à tester l'impact de quatre types de scénarios concernant la pluviométrie d'hivernage :

- les producteurs n'ont aucune information sur l'état de la saison à venir ;

- les producteurs ont accès à une prévision de saison sèche ;

- les producteurs ont accès à une prévision de saison normale ;

- les producteurs ont accès à une prévision de saison humide. 
On considère que les producteurs n'ont pas de difficulté à interpréter l'information climatique et que toute information conduit à un changement d'assolement. Dans le modèle, pour rendre compte d'un scénario sans prévision, on attribue des probabilités identiques (33 p. 100) de distribution de chacun des trois types d'hivernage (humide, normal, sec) qui est la manière utilisée par l'African Centre of Meteorological Application for Development pour présenter les prévisions en Afrique de l'Ouest. Cela signifie en d'autres termes que chaque type d'hivernage à la même probabilité d'occurrence. Nous avons construit les autres scénarios en attribuant une probabilité plus élevée de 80 p. 100 au type d'hivernage prédit par la prévision. Les probabilités associées à chaque scénario sont présentées dans le tableau I.

Pour un scénario donné, le modèle calcule l'assolement, puis le revenu associé à chaque type d'hivernage que multiplie son coefficient respectif. Puis, il réalise la somme des trois revenus. En cas de scénario de prévision de saison humide, le modèle va choisir un assolement constitué de cultures présentant les meilleurs rendements dans ce contexte-là, comme le maïs et le sorgho. Quand la prévision est sèche, le modèle va favoriser le mil, culture présentant les meilleurs rendements lorsque l'hivernage est sec.

Nous présentons l'impact de ces prévisions sur l'assolement des cultures, le revenu monétaire et les valeurs marginales du travail, de la terre et de l'eau. Ce qui se passe en cas d'erreur de prévision a également été analysé.

Pour simuler une erreur de prévision, quand par exemple la saison est humide alors qu'elle était prévue sèche, le modèle calcule le revenu en multipliant l'assolement calculé pour l'hivernage sec par les rendements et les prix de l'hivernage humide. Le revenu est alors plus faible que si la prévision était bonne.

Tableau I

Probabilités des prévisions saisonnières dans les quatre scénarios

\begin{tabular}{lccc|} 
Scénario & \multicolumn{3}{c}{ Type d'hivernage } \\
\cline { 2 - 4 } & $\begin{array}{c}\text { Hivernage } \\
\text { sec }\end{array}$ & $\begin{array}{c}\text { Hivernage } \\
\text { normal }\end{array}$ & $\begin{array}{c}\text { Hivernage } \\
\text { humide }\end{array}$ \\
\hline Pas de prévision & 33 & 33 & 33 \\
Prévision sèche & 80 & 10 & 10 \\
Prévision normale & 10 & 80 & 10 \\
Prévision humide & 10 & 10 & 80
\end{tabular}

\section{RESULTATS}

\section{Allocation des terres}

Les résultats du scénario sans prévision montrent que les terres disponibles pour les cultures pluviales sont cultivées aux deux tiers (figure 2), le reste, et en particulier les terres marginales, étant mis en jachère. Les cultures pluviales sont surtout le sorgho et le coton sur les bonnes terres, le mil sur les terres marginales, le maïs et le riz dans les bas-fonds peu inondés, ce qui est un assolement proche de la réalité. Les parcelles irriguées sont allouées à la production de riz pendant l'hivernage, puis à la tomate pendant la saison sèche froide et pendant la saison sèche chaude mais sur une moindre surface pour cette dernière période, le reste étant mis en jachère.

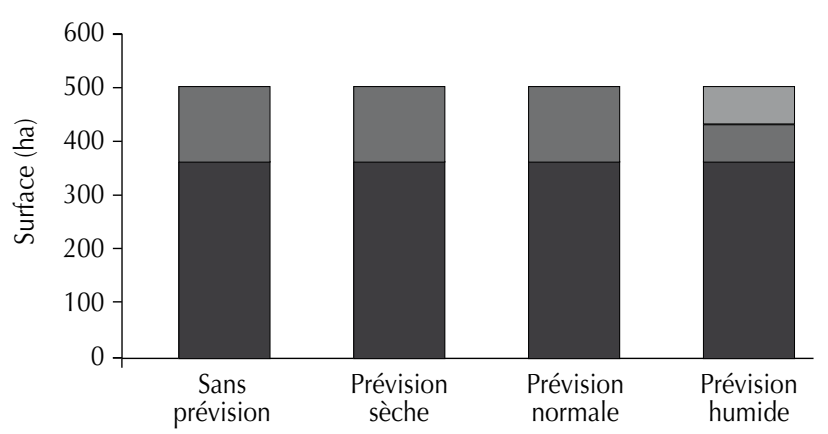

A $\square$ Sorgho terres marginales

$\square$ Mil terres marginales

Jachères terres marginales

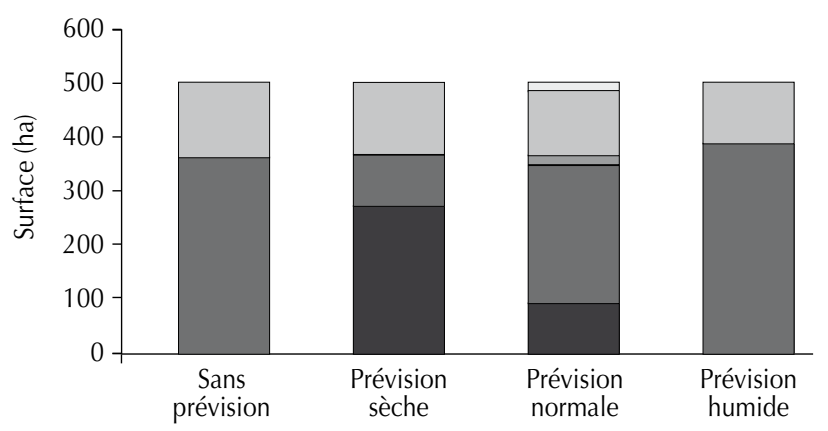

B $\square$ Maïs intensif bonnes terres $\square$ Sorgho bonnes terres Mil bonnes terres

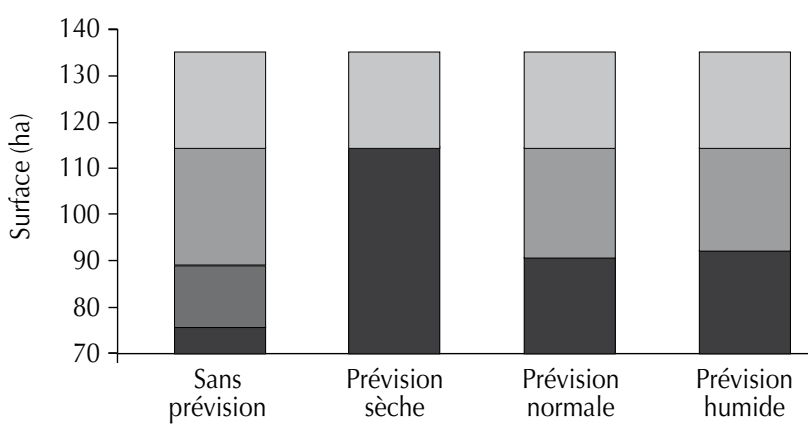

C $\square$ Maïs intensif bas-fond peu inondé $\square$ Riz bas-fond inondé $\square$ Maïs extensif bas-fond peu inondé $\square$ Riz bas-fond peu inondé

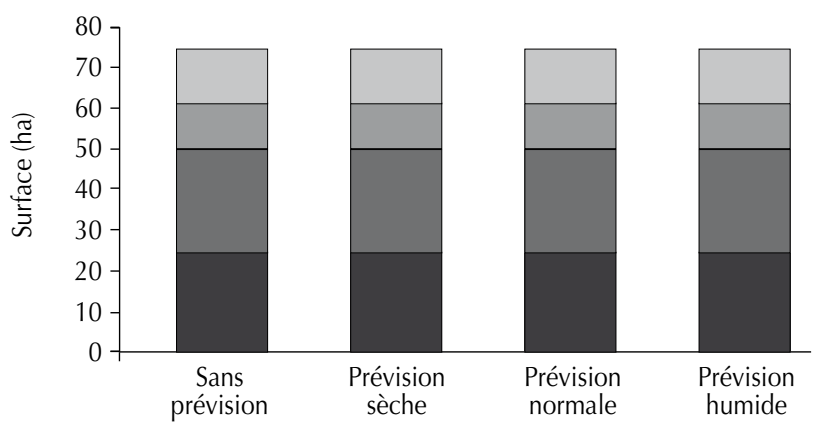

D $\square$ Tomate irrigué saison froide $\square$ Jachère saison chaude $\square$ Riz irrigué $\square$ Tomate irrigué saison chaude

Figure 2 : allocation optimale des terres pour les différents scénarios ( $A$ : terres marginales ; $B$ : bonnes terres ; $C$ : basfonds ; $D$ : terres irriguées). 
Si la prévision est un hivernage sec, le mil est non seulement cultivé sur les terres marginales, mais aussi sur les bonnes terres au détriment du sorgho. Le maïs est exclusivement cultivé dans les bas-fonds peu inondés au détriment du riz. L'assolement sur les parcelles de bas-fonds inondés et sur les parcelles irriguées est identique à celui du scénario sans prévision.

Dans le scénario prévoyant un hivernage normal, le sorgho est réintroduit sur les bonnes terres, de même que le maïs sur une petite surface, le riz réapparaît dans les bas-fonds peu inondés ainsi qu'une petite surface de maïs très intensif. L'allocation de la terre reste inchangée pour les bas-fonds inondés et les parcelles irriguées.

Pour le scénario de prévision d'un hivernage humide, le sorgho reprend totalement la place du mil sur les bonnes terres comme pour le scénario sans prévision. Le sorgho est aussi cultivé sur les terres marginales au détriment du mil réduit de moitié. L'allocation de la terre reste inchangée pour les cultures irriguées, celle des bas-fonds peu inondés est quasiment identique à celle du scénario sans prévision.

En résumé, en cas de prévision d'hivernage sec, le modèle favorise la culture de mil, mais il privilégie le sorgho et le maïs en cas de prévision d'hivernage humide.

\section{Revenus monétaires}

Lorsque le modèle ne dispose pas de prévisions sur l'hivernage et que l'hivernage est normal, le revenu optimal du bassin est de 113 millions de francs CFA (figure 3), si l'hivernage s'avère plutôt sec, le revenu baisse de 24 p. 100 à cause de la baisse des rendements ; s'il est humide, il augmente de 17 p. 100.

Lorsque les producteurs disposent d'une prévision d'année sèche et que l'année est effectivement sèche, on observe une hausse de 8 p. 100 du revenu simulé par rapport à l'année sèche de la simulation sans prévision. En revanche, si l'année est normale, le revenu simulé baisse de 10 p. 100 par rapport à l'année normale sans prévision alors que, si elle est humide, il baisse de 16 p. 100 par rapport à l'année humide sans prévision. Les gains de la prévision sont conséquents mais les pertes en cas d'erreur sont supérieures.

Lorsque les producteurs ont une prévision d'année normale et que l'hivernage est effectivement normal le revenu n'augmente que de 2 p. 100 par rapport à l'hivernage normal de la simulation sans prévision. Mais si l'hivernage s'avère sec, le revenu simulé ne baisse pas par rapport à l'année sèche simulée sous le scénario sans prévision. En revanche, si l'année s'avère humide, le revenu

$\square$ Hivernage sec $\quad \square$ Hivernage normal $\quad \square$ Hivernage humide

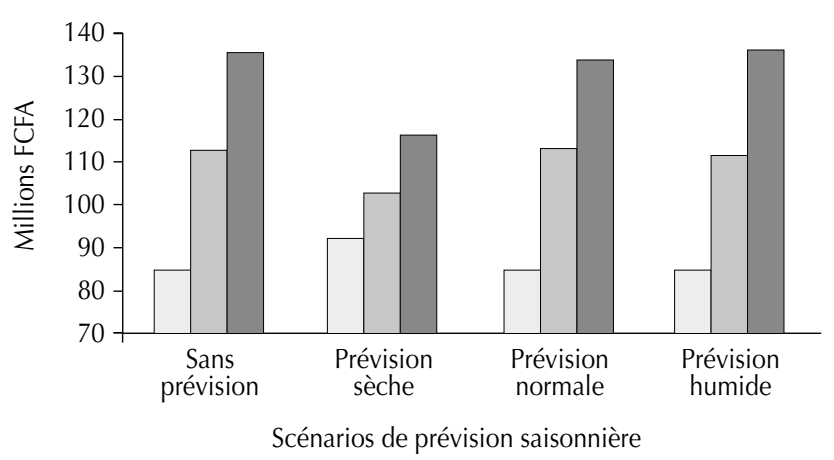

Figure 3 : revenus selon les prévisions saisonnières et leur réalisation. diminue de 5 p. 100 par rapport à l'année humide simulée sous le scénario sans prévision.

Lorsque les producteurs ont une prévision d'année humide et que l'année est effectivement humide le revenu n'augmente presque pas par rapport à l'année humide simulée sous le scénario sans prévision. Si l'année s'avère normale le revenu diminue de 1 p. 100 par rapport à l'année normale sous le scénario sans prévision. Si l'année s'avère sèche le revenu ne change pas par rapport à l'année sèche sous le scénario sans prévision.

En somme, les différences sont surtout importantes en prévision d'une saison sèche car le modèle choisit alors des cultures comme le mil, au détriment du sorgho et du maïs aux rendements potentiels supérieurs. Pour les scénarios de prévision d'hivernage normal ou humide, le gain d'une bonne prévision est nul ainsi que le coût d'une forte erreur de prévision parce que le modèle est calibré pour ne pas descendre en dessous d'un revenu minimum en cas de mauvaise année. En effet, le modèle ne peut investir que de façon limitée dans des cultures plus productives mais plus risquées en raison d'une chute importante de leur rendement en cas de mauvaise année.

\section{Valeurs marginales}

Les valeurs marginales des facteurs terre, travail, capital et risque indiquent l'importance de la rareté d'un facteur. Si l'on compare une valeur marginale aux prix du marché local pour ces facteurs on peut identifier les facteurs les plus contraignants et faire des propositions en termes de politique agricole. Pour la contrainte de travail, seule la période d'installation des cultures pluviales en juin/juillet a une valeur marginale qui est de 2000 FCFA par jour (tableau II). Cela signifie que si les paysans pouvaient sur un plan théorique travailler une journée de plus et sans tenir compte de facteurs sociaux, le gain journalier pour le ménage serait de 2000 FCFA. Cette valeur est localement trois fois supérieure au coût d'une journée de travail par actif. Elle reflète le manque de disponibilité de la main d'œuvre. La main d'œuvre est donc un facteur contraignant pour la production, ce qui se traduit par la mise en jachère d'une partie des terres pluviales du village. Cela veut aussi dire que les producteurs du bassin gagneraient à embaucher de la main d'œuvre extérieure à condition de la rémunérer à un prix inférieur à la valeur marginale.

Les différences constatées entre les types de prévision sont importantes, ce qui traduit le fait qu'une prévision favorable et fiable augmenterait la productivité du travail.

La valeur marginale de la terre représente ce que gagnerait un producteur s'il pouvait augmenter d'un hectare la disponibilité des terres pluviales, irriguées ou de bas-fond (tableau II). Les terres marginales en pluvial ne sont pas encore contraignantes puisqu'une fraction importante de ces terres est mise en jachère, tous scénarios confondus. En revanche, les autres types de terres sont contraignants, et cela d'autant plus que leur surface est limitée par rapport à la surface totale cultivable. La surface irriguée est entièrement utilisée pour les deux premières campagnes irriguées mais pas pour la troisième par manque d'eau dans le barrage en cas d'hivernage sec. La valeur marginale très élevée des terres irriguées s'explique, en outre, par le fait que le modèle est averse au risque. En effet, un hectare irrigué en plus permettrait d'augmenter la surface de cultures irriguées et d'investir dans des cultures plus productives sans prendre de risque en cas de mauvaise année. Le capital est limitant car entièrement utilisé. En moyenne, un franc CFA investi augmenterait le revenu net final de 0,20 FCFA. C'est un retour sur investissement de 20 p. 100. 


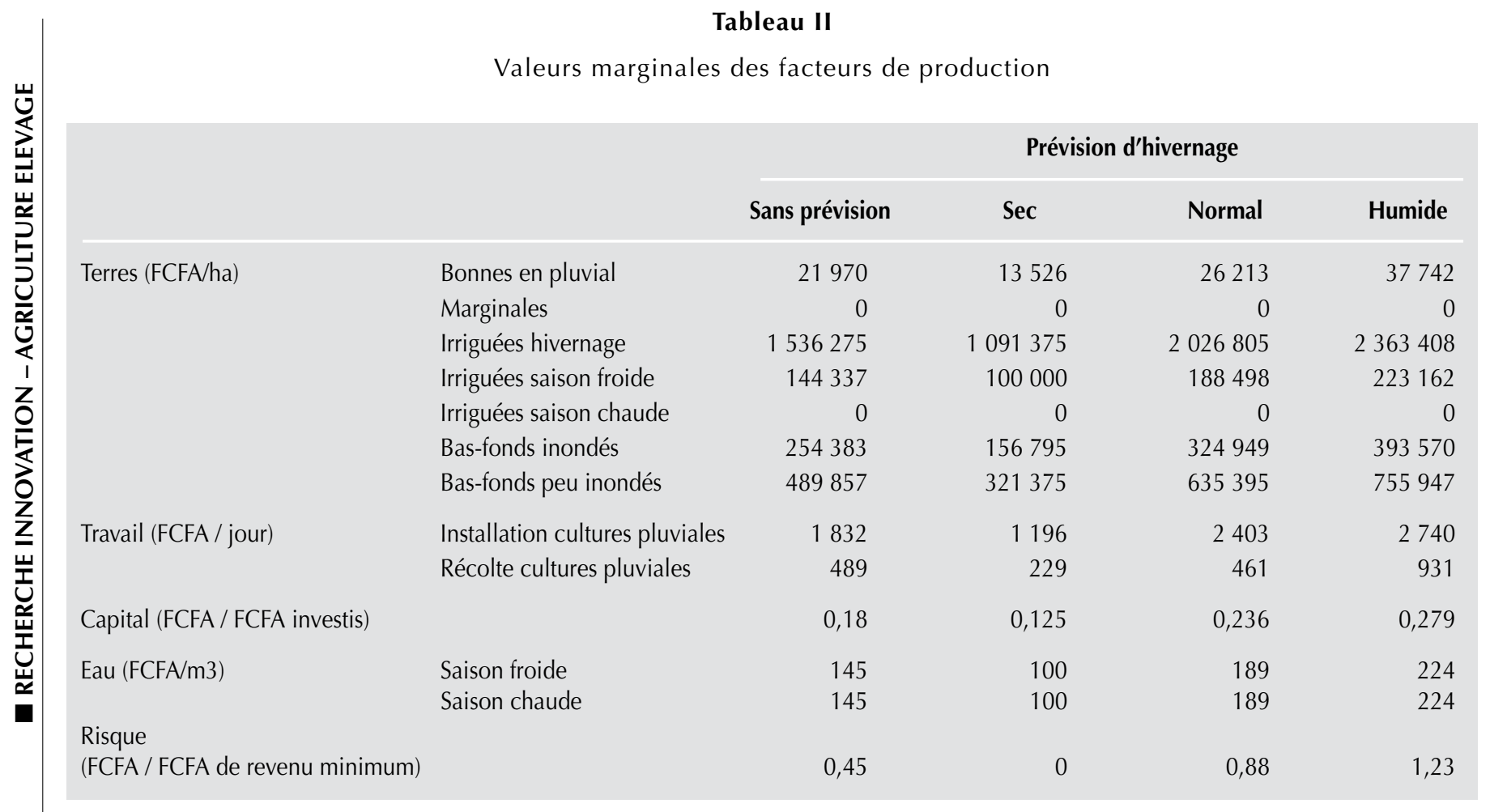

L'eau d'irrigation est un facteur limitant pour les cultures irriguées pour les deux campagnes de contre-saison. Les valeurs sont les mêmes entre les deux périodes car l'eau non utilisée pendant une période est reportée à la période suivante. Le mètre cube d'eau supplémentaire rapporterait 145 FCFA dans le scénario sans prévision saisonnière. Ce gain augmente si la prévision est humide du fait d'un revenu légèrement supérieur.

Nous avons utilisé un revenu minimum pour paramétrer le modèle afin de reproduire l'assolement que les paysans pratiquent réellement dans la zone. La valeur marginale de la contrainte de risque signifie que, si les paysans acceptaient de réduire leur revenu minimal en cas de mauvaise année, ils pourraient augmenter leur revenu global les années normales et humides. Un franc CFA de revenu minimum en moins augmenterait le revenu de 45 centimes pour le scénario sans prévision et jusqu'à un franc CFA et 23 centimes pour un scénario de prévision d'hivernage humide. Dans ce dernier cas, la différence est maximale entre le revenu que peut obtenir le modèle et le revenu minimum. La prime de risque d'une assurance doit être strictement inférieure au montant de la valeur marginale pour être intéressante pour le producteur.

\section{DISCUSSION}

\section{Contraintes du système}

Les valeurs marginales montrent que tous les facteurs de production du modèle sont contraignants : le travail en début d'hivernage et à la récolte, certains types de terre, le capital et l'eau. Le travail est contraignant dans la mesure où toutes les terres marginales ne sont pas mises en culture. De plus, la consommation en grains est satisfaite et le surplus monétaire par personne important (56 000 FCFA) même les mauvaises années (44 000 FCFA). L'eau est un facteur limitant puisque le barrage est petit et que toute l'eau est utilisée pour les rizières et le maraîchage. Le risque est également limitant puisque les paysans cultivent encore du mil pour éviter l'impact d'une sécheresse, diminuant ainsi le revenu moyen. Or la zone sud-ouest du Burkina Faso est une zone à fort potentiel où il est possible d'introduire des cultures pouvant obtenir de meilleurs rendements.

Le capital est très limitant, même si le modèle donne la possibilité de contracter du crédit lié à la production de coton. Le modèle introduit donc une surface de coton uniquement pour que les producteurs aient accès au crédit. Les sociétés cotonnières assurent une partie du financement des campagnes mais ne peuvent pas financer les besoins des cultures non cotonnières. Le système bancaire agricole fonctionne mal, faute de garantie bancaire. Il faudra attendre qu'un marché de la terre émerge pour fournir les garanties bancaires nécessaires et voir la contrainte de crédit évoluer. D'où l'intérêt d'autres systèmes de garantie, telle que la caution solidaire proposée par les sociétés cotonnières. Un moyen d'améliorer l'octroi de crédit bancaire est de réduire le risque agricole, qui réduirait le risque de non-remboursement. Pour ce faire la prévision saisonnière est une option.

\section{Prévisions saisonnières}

Des deux hypothèses formulées dans cette étude, la première était que le gain des prévisions était surtout intéressant pour une prévision d'hivernage humide. Cette hypothèse a été infirmée. Le gain de la prévision a été important lorsqu'elle a concerné une année sèche. Le gain d'une prévision a été faible pour une prévision d'hivernage humide. Ce résultat a été lié au choix de calibrer le modèle pour ne pas descendre en dessous d'un revenu minimum, ce choix se basant sur les stratégies observées dans la zone. En effet, les paysans faisaient référence à un revenu minimal à atteindre pour rembourser notamment le crédit attribué par les sociétés cotonnières. Une prévision d'hivernage humide ou normal ne changeait pas grand chose au choix des cultures et au revenu.

La seconde hypothèse était que le coût d'une erreur de prévision humide était plus important que le gain d'une bonne prévision. Cette hypothèse n'a été confirmée que lorsqu'une année prévue sèche s'est avérée humide ou lorsqu'une année humide s'est avérée sèche, soit lors de très grandes erreurs. Le coût d'une erreur de prévision d'une année humide lorsque l'année s'est avérée 
normale a été faible. Il faudrait ainsi envisager un système d'assurance. Par ailleurs, l'irrigation a été peu influencée par la variabilité climatique pour les périmètres situés en aval de barrages. En effet, les surfaces sont restées les mêmes quelle qu'ait été la prévision saisonnière.

\section{Adoption des prévisions saisonnières par les populations}

Nous avons considéré dans cette étude que les producteurs n'avaient pas de difficulté à interpréter l'information climatique. La réalité peut être différente. Les prévisions saisonnières pour être promues doivent gagner en fiabilité mais surtout doivent faire l'objet d'un accompagnement préalable dans des contextes où la majorité des producteurs ne sont pas instruits. Les producteurs doivent être formés sur la façon d'interpréter les résultats des prévisions et celles-ci doivent leur être livrées avec des informations sur les marges d'erreur.

Cette étude a permis d'analyser l'avantage relatif (8) de la prévision saisonnière par rapport aux pratiques existantes et à différentes modalités de prévision saisonnière, en analysant les coûts et risques associés mais aussi les valeurs marginales du modèle. Les modèles d'optimisation, comme celui présenté dans cette étude, sont de puissants outils d'évaluation ex ante $(7,12)$ permettant de façon virtuelle de tester la proposition. Pour ce type de modèles, la prise en compte de différents objectifs $(2,6)$ ou de contraintes environnementales et des besoins de sécurité alimentaires, comme c'est le cas ici, permet de tenir compte de stratégies de production qui ne sont pas seulement basées sur la maximisation du revenu.

Mais comme tout modèle informatisé, l'une des difficultés rencontrées est l'accès à des données fiables pour renseigner les paramètres (13) et cela en particulier dans des contextes où les producteurs n'enregistrent pas leurs pratiques. Dans ce modèle, nous nous sommes focalisés sur les systèmes de cultures pour lesquels la prévision saisonnière apparaissait plus pertinente. Néanmoins, ce modèle pourrait être amélioré de façon à simuler les troupeaux de petits ruminants qui, bien que limités, peuvent jouer un rôle non négligeable d'épargne dans ces systèmes. Ce modèle amélioré pourrait en particulier permettre d'affiner l'analyse du coût marginal du capital ou du risque.

\section{BIBLIOGRAPHIE}

1. ADESINA A.A.A., OUATTARA A.D., 2000. Risk and agricultural systems in northern Côte d'lvoire. Agric. Syst., 66: 17-32.

2. DOGLIOTTI S., ITTERSUM M.K.V., ROSSING W.A.H., 2005. A method for exploring sustainable development options at farm scale: a case study for vegetable farms in South Uruguay. Agric. Syst., 86: 29-51.

3. INGRAM K.T., RONCOLI M.C., KIRSHEN P.H., 2002. Opportunities and constraints for farmers of West Africa to use seasonal precipitation forecasts with Burkina Faso as a case study. Agric. Syst.,74: 331-349.

4. JANNOT P., CAIROL D., 1994. Linear programming as an aid to decision making for investments in farm equipment for arable farms. J. Agric. Engin. Res., 59: 173-179.

5. MAURIZIO B., LORENZO G., ANDREA D.V., IDRISSA A., BIRAMA D., MAMADOU N., 2008. Les prévisions saisonnières et leurs impact sur la prévention de l'insécurité alimentaire : le cas d'étude de la campagne $2007 / 2008$ et la prévision pour la campagne 2008/2009. In: Seasonal forecasting in West Africa, its applications and anticipating future climate change, AMMA/ENSEMBLES workshop, Niamey, Niger, 26-30 May 2008.

6. OKORUWA V., JABBAR M.A., AKINWUMI J.A., 1996. Crop-livestock competition in the West African derived savanna: Application of a multiobjective programming model. Agric. Syst., 52 : 439-453.

7. PACINI C., WOSSINK A., GIESEN G., HUIRNE R., 2004. Ecologicaleconomic modelling to support multi-objective policy making: a farming systems approach implemented for Tuscany. Agric. Ecosyst. Environ., 102: 349-364.
8. SATTLER C., NAGEL U.J., 2010. Factors affecting farmers' acceptance of conservation measures. A case study from North-Eastern Germany. Land Use Policy, 27: 70-77.

9. SHARDUL A., FRANKHAUSER S., 2008. aspects économiques de I'adaptation aux changements climatiques: coût, bénéfices et instruments économiques. Paris, France, OECD, 9 p.

10. SULTAN B., BARBIER B., FORTILUS J., MODOU MBAYE S. LECLERC G., 2010. Estimating the potential economic value of seasonal forecasts in West Africa: A long-term ex-ante assessment in Senegal. Weather climate Soc., 2: 69-87. http://dx.doi. org/10.1175/2009WCAS1022.1 Consulté le 27.09.2010

11. THOMAS D., TWYMAN C., OSBAHR H., HEWITSON B., 2007. Adaptation to climate change and variability: farmers' responses to intraseasonal precipitation trends in South Africa. Clim. Change, 83: 301-322.

12. TORKAMANI J., 2005. Using a whole-farm modelling approach to assess prospective technologies under uncertainty. Agric. Syst ., 85: 138-154.

13. VAN WIJK M.T., TITTONELL P., RUFINO M.C., HERRERO M. PACINI C., RIDDER N.D., GILLER K.E., 2009. Identifying key entrypoints for strategic management of smallholder farming systems in sub-Saharan Africa using the dynamic farm-scale simulation model NUANCES-FARMSIM. Agric. Syst., 102: 89-101.

14. YILI T., 2006. La monographie de la commune rurale de Dano. Ouagadougou, Burkina Faso, ministère de l'Economie et du Développement, $62 \mathrm{p}$.

Mis en ligne en juin 2013 


\section{Summary}

Dabire W.P.I., Barbier B., Andrieu N. Ex ante assessment of climatic seasonal predictions for small-scale farming in Burkina Faso

Agricultural production in sub-Saharan Africa is mostly rainfed and particularly vulnerable to climatic variations. At the scale of the farm, climatic variability has a direct impact on primary production and on the household's income and food security. In this study, we assessed the economic benefit of providing rainy season rainfall predictions to producers. The study was carried out in the south-west of Burkina Faso, in the area of Dano. It is based on a linear programming model that maximizes income by optimizing the allocation of land, labor and inputs among several crops, with yields varying in relation to the type of soil and crop management sequence, and to the rainy season rainfall pattern. Four scenarios were analyzed, differing according to the type of information available to farmers concerning the oncoming rainy season rainfall: a control scenario with no forecast given, a low-rainfall rainy season forecast, a normal rainy season forecast, and a wet rainy season forecast. The results of the simulations show that the most important prediction is the one warning of a dry rainy season, but this is also the one for which prediction errors entail the most costly consequences. Overall, the income gain associated with predictions is relatively small, but the cost incurred by a prediction error raises the issue of liability and compensation. These results shed a sobering light on the value of seasonal predictions for alleviating the vulnerability of Sahel communities.

Keywords: Linear programming - Decision support - Season Forecasting - Pluviometry - Climate - Risk management Burkina Faso.

\section{Resumen}

Dabire W.P.I., Barbier B., Andrieu N. Evaluación ex ante de la previsión estacional climática en un pequeño campesinado burkinés

La producción agrícola de Africa sub sahariana, esencialmente pluvial, es particularmente vulnerable a la variabilidad climática. A nivel de las explotaciones, esta variabilidad tiene un impacto directo sobre la producción primaria, así como sobre el ingreso y la seguridad alimenticia de la familia. En el presente estudio, nosotros evaluamos el interés económico de poner a la disposición de los productores la previsión de la pluviometría de invierno. El estudio se llevó a cabo en la zona de Dano, al suroeste de Burkina Faso. Este reposa sobre la utilización de un modelo de programación linear que maximiza el ingreso, optimizando la asignación de las tierras, del trabajo y de los ingresos entre varios tipos de cultivos, los rendimientos varían según el tipo de suelo, el itinerario técnico y el invierno. Se analizaron cuatro escenarios: un escenario testigo en donde el campesino no tiene acceso a las previsiones, un escenario en donde los productores tienen acceso a una previsión de invierno seco, otro donde tienen acceso a una previsión de invierno normal y finalmente un último en donde tienen acceso a una previsión de invierno húmedo. Los resultados de estas simulaciones muestran que es la previsión de invierno seco la que presenta el mayor interés, pero es también en la que un error de previsión sería mas costoso. Globalmente la ganancia en ingreso de la previsión es relativamente poco elevada, pero el costo de un error de previsión presenta el problema de las responsabilidades y de las compensaciones. Estos resultados relativizan el interés de las previsiones estacionales para la reducción de la vulnerabilidad de las poblaciones de los países del Sahel.

Palabras clave: Programación lineal - Soporte de decisión Estación del año - Pronóstico - Pluviometría - Clima - Gestión de riesgos - Burkina Faso. 